\title{
Second-harmonic generation microscopy analysis reveals proteoglycan decorin is necessary for proper collagen organization in prostate
}

Kirby R. Campbell Rajeev Chaudhary Monica Montano Renato V. Iozzo

Wade A. Bushman Paul J. Campagnola 


\title{
Second-harmonic generation microscopy analysis reveals proteoglycan decorin is necessary for proper collagen organization in prostate
}

\author{
Kirby R. Campbell, ${ }^{\mathrm{a}}$ Rajeev Chaudhary, ${ }^{\mathrm{a}}$ Monica Montano, ${ }^{\mathrm{b}}$ Renato V. lozzo, ${ }^{\mathrm{c}}$ Wade A. Bushman, ${ }^{\mathrm{b}}$ and \\ Paul J. Campagnola ${ }^{a, *}$ \\ aUniversity of Wisconsin-Madison, Department of Biomedical Engineering, Madison, Wisconsin, United States \\ bUniversity of Wisconsin-Madison, Department of Urology, Madison, Wisconsin, United States \\ ${ }^{c}$ Thomas Jefferson University, Department of Pathology, Philadelphia, Pennsylvania, United States
}

\begin{abstract}
Collagen remodeling occurs in many prostate pathologies; however, the underlying structural architecture in both normal and diseased prostatic tissues is largely unexplored. Here, we use second-harmonic generation (SHG) microscopy to specifically probe the role of the proteoglycan decorin (Dcn) on collagen assembly in a wild type (wt) and Dcn null mouse ( $\left.\mathrm{Dcn}^{-/}\right)$. Dcn is required for proper organization of collagen fibrils as it regulates size by forming an arch-like structure at the end of the fibril. We have utilized SHG metrics based on emission directionality (forward-backward ratio) and relative conversion efficiency, which are both related to the SHG coherence length, and found more disordered fibril organization in the $\mathrm{Dcn}^{-/-}$. We have also used image analysis readouts based on entropy, multifractal dimension, and wavelet transforms to compare the collagen fibril/fiber architecture in the two models, where all these showed that the $\mathrm{Dcn}^{-/-}$prostate comprised smaller and more disorganized collagen structures. All these SHG metrics are consistent with decreased SHG phase matching in the $\mathrm{Dcn}^{-/-}$and are further consistent with ultrastructural analysis of collagen in this model in other tissues, which show a more random distribution of fibril sizes and their packing into fibers. As Dcn is a known tumor suppressor, this work forms the basis for future studies of collagen remodeling in both malignant and benign prostate disease. () The Authors. Published by SPIE under a Creative Commons Attribution 4.0 Unported License. Distribution or reproduction of this work in whole or in part requires full attribution of the original publication, including its DOI. [DOI: 10.1117/1.JBO.24.6.066501]
\end{abstract}

Keywords: prostate; collagen second-harmonic generation; image analysis; coherence.

Paper 190005R received Jan. 8, 2019; accepted for publication Apr. 18, 2019; published online May $30,2019$.

\section{Introduction}

Proper collagen organization is critical for normal function and the architecture becomes abnormally assembled in many diseases, including connective tissue disorders, cancers, and fibroses. To use these alterations as biomarkers, it is critical to know the proper assembly in normal tissues to be used as comparison for diagnostic purposes. Alterations can be in the form of increased collagen synthesis, misformed collagen, differential isoform expression, and/or increase or loss of related matrix proteins. For example, in the connective tissue disorder osteogenesis imperfecta, the collagen is misfolded and results in incorrect fibril size and spacing, smaller fibers, and weaker mechanical structures. ${ }^{1-3}$ Relatedly, the loss of $\mathrm{Col} \mathrm{V}$, which is essential for correct Col I fibril formation, results in numerous structural defects. ${ }^{4,5}$ Moreover, an increase in Col V and III have been associated with cancers and fibroses in several tissues. ${ }^{6-8}$

In this paper, we examine the effect of loss of the structural protein decorin on collagen assembly in the prostate, where we specifically quantify differences by using several secondharmonic generation (SHG) metrics developed in our laboratory. ${ }^{9}$ Although there have been a few reports on using SHG to image prostate cancer and benign prostate hyperplasia, ${ }^{10-12}$ the underlying collagen structure (fibril and fiber assembly) in the prostate has not been well examined. This

*Address all correspondence to Paul J. Campagnola, E-mail: pcampagnola@ wisc.edu is a significant consideration as such studies are necessary for better eventual diagnostics. To this end, we use a wellestablished mouse model $\left(\mathrm{Dcn}^{-/-}\right)^{13}$ that has systemic loss of decorin. In addition to better understanding the role of Dcn on normal prostate assembly, insight into the consequences of its loss is important as it has been implicated as a tumor suppressor of prostate cancer. ${ }^{14}$ Moreover, the $\mathrm{Dcn}^{-/-}$mice are deficient in autophagy and more prone to develop tumors in other tissues studied previously. ${ }^{15-18}$

The mouse prostate is a male accessory sex organ that comprises an exocrine ductal network enmeshed in a supporting stroma and divided into three distinct lobes: the coagulating gland [also known as the anterior prostate (AP)], dorsolateral prostate, and ventral prostate (VP). The prostate develops from the urogenital sinus in response to androgen stimulation and depends on a variety of signaling interactions involving a variety of factors, including sonic and Indian hedgehog ( $\mathrm{SHH}$ and IHH), fibroblast growth factor 10 (FGF10), bone morphogenetic proteins 4 and 7, transforming growth factor $\beta$, notch1, nk3 homeobox 1, and forkhead box a1. These factors promote epithelial proliferation and prostatic bud initiation, elongation, and branching morphogenesis. ${ }^{19,20}$

Decorin is an integral structural component of the extracellular matrix that binds to collagen fibrils and influences collagen organization. In normal tissue, the Dcn molecule acts as an arch around the fibrils, leading to relatively uniform fibril size and spacing. ${ }^{21-23}$ Den is colocalized with collagen in the periductal stroma and acellular interstitium and exerts a growth inhibitory 
effect on epithelial proliferation. Studies examining various tissues, such as the skin, bone, and heart, have shown that loss of Dcn results in irregular fibril diameter and abnormal fibrillar organization. ${ }^{13,24-26}$ These findings suggest that loss of Den might incur similar disruptions in the architecture of the prostate, but this has not been previously investigated.

We have previously shown how SHG microscopy is a powerful tool for analyzing changes in collagen organization in many diseased states, including ovarian cancer, colon cancer, pulmonary fibrosis, and connective tissue disorders. ${ }^{27-30}$ Here, we use SHG microscopy to examine the collagen fibril and fiber architecture in the normal developing mouse prostate and $\mathrm{Dcn}^{-/-}$ prostatic collagen matrix. We will specifically use our previously established tools in analyzing the SHG emission directionality, SHG efficiency in three-dimensional (3-D) tissues, ${ }^{9}$ as well as use wavelet transform analyses to analyze the fiber patterns in the wild type (wt) and $\mathrm{Dcn}^{-/-}$prostate tissues. All these tools exploit the differences in the SHG coherence properties that result from the normal and $\mathrm{Dcn}^{-/-}$matrices, specifically the difference in fibril structure that underlies the SHG contrast. ${ }^{31}$ Importantly, all the SHG analyses are consistent with known ultrastructural biology assessments of the role of $\mathrm{Dcn}^{-/-}$in other tissues, ${ }^{22,23}$ while being performed on intact tissue slices on the SHG microscope.

\section{Experimental Methods}

\subsection{Animals}

$\mathrm{Dcn}^{-/-}$mice were provided by Dr. Renato Iozzo (Thomas Jefferson University, Philadelphia, Pennsylvania). All mice were maintained on a 12-h light and dark cycle. All procedures were approved by the University of Wisconsin Animal Care and Use Committee. Adult ( 8 to 10 weeks) $\mathrm{Dcn}^{-/-}$and wt (+/+) littermates were used in all studies. Genotypes were confirmed using PCR analysis. ${ }^{13}$

\subsection{Tissue Preparation}

Prostates were obtained from $\mathrm{Dcn}^{-/-}$and wt male mice. The mice were anesthetized, sacrificed, and individual prostatic lobes were dissected. All prostatic lobes were immediately fixed in $4 \%$ formalin and refrigerated for $24 \mathrm{~h}$ before being switched to phosphate-buffered saline. The specimens were sectioned using a Leica Vibratome 1200S (Leica Biosystems, Buffalo Grove, Illinois) to thicknesses of $100 \mu \mathrm{m}$ for SHG imaging. SHG imaging was performed on both the AP and VP lobes (from three wt and $\mathrm{Dcn}^{-/-}$mice), and at least three $z$ series of $\sim 100 \mu \mathrm{m}$ in thickness were imaged per tissue. We have previously shown that fixing does not affect the resulting fibrillar assembly and only results in a slight shrinking of the tissue. ${ }^{32}$

\subsection{SHG Imaging System}

The SHG system used for this study has been previously described. ${ }^{9}$ A mode-locked titanium sapphire laser excitation source (coherent Mira, Santa Clara, California) with a pulse width and repetition rate of $\sim 100$ fs and $\sim 80 \mathrm{MHz}$, respectively, was coupled to a laser scanning unit (FluoView 300; Olympus, Melville, New York) mounted on an upright microscope (BX61; Olympus). All imaging was done at an excitation wavelength of $890 \mathrm{~nm}$ with an average power of $\sim 20 \mathrm{~mW}$ at the specimen using a water immersion $40 \times 0.8 \mathrm{NA}$ objective. This system provided lateral and axial resolutions of $\sim 0.7$ and $2.5 \mu \mathrm{m}$, respectively.

The microscope simultaneously collected both the forward (F) and backward (B) components of the SHG intensity using identical calibrated detectors $(7421$ GaAsP photon counting modules; Hamamatsu, Hamamatsu City, Japan). The SHG wavelength $(445 \mathrm{~nm})$ was isolated with a $10-\mathrm{nm}$-wide bandpass filter (Semrock, Rochester, New York). Calibration of the F and B detection pathways was performed using two-photon excited fluorescence imaging of polystyrene beads emitting $445 \mathrm{~nm}$. Circularly polarized light was verified at the focus to ensure equal excitation of all fiber orientations. ${ }^{9}$

For all imaging metrics, we chose fields where there was at least $60 \%$ collagen coverage. This is in accordance with our previously published analyses in other tissues, e.g., ovarian tissues using SHG F/B and attenuation measurements as well as texture analyses..$^{29,33}$

\subsection{Image Analysis}

\subsubsection{SHG emission directional analysis}

We determine the $\mathrm{SHG}$ directional emission ratio $\mathrm{F}_{\mathrm{SHG}} / \mathrm{B}_{\mathrm{SHG}}$ (or SHG creation ratio) which is reflective of the fibril diameter, the packing density, and regularity relative to the size scale of the SHG wavelength. ${ }^{31}$ This is determined by first measuring the $F / \mathrm{B}$ intensity ratio versus depth, where this axial response arises from a convolution of $F_{\mathrm{SHG}} / B_{\mathrm{SHG}}$ and subsequent propagation through the tissue which is based on the scattering coefficient $\left(\mu_{s}\right)$ and scattering anisotropy $(g)$ at the SHG wavelength. ${ }^{30}$ The measured SHG directional (F/B ratios) values were determined per optical section every $10 \mu \mathrm{m}$ of depth using MATLAB software (Mathworks, Natick, Massachusetts). Monte Carlo simulations based on our modified customized MCML code ${ }^{34}$ for SHG microscopy ${ }^{30,35}$ are then used to uniquely extract $F_{\mathrm{SHG}} / B_{\mathrm{SHG}}$ using the scattering coefficients of prostate. ${ }^{36}$ In the treatment here we used values from the literature, where the $\mu_{s}$ is assumed to be $445 \mathrm{~cm}^{-1}$ and $g$ to be 0.93 .

\subsubsection{SHG conversion efficiency analysis}

We determine the relative SHG conversion efficiencies from each tissue. This metric is a convolved effect of the collagen concentration (square thereof) and the fibrillar organization relative to the wavelength of SHG emitted light ${ }^{37}$ and results from the magnitude of the second-order nonlinear susceptibility tensor $\chi^{(2)}$ and phase-matching conditions. ${ }^{38}$ Specifically, better phase matching results in brighter SHG. The relative conversion efficiency is determined by measuring the forward attenuation, i.e., relative SHG intensity as a function of depth through the tissue. Normalization between tissues is necessary to account for local variability within the same tissue (different fields of view) and to make relative comparisons between tissues. ${ }^{30}$ This metric is dominated by the conversion efficiency and primary filter (squared) based on $\mu_{s}$ at the excitation wavelength. Our SHG MCML framework is then used to decouple the conversion efficiency. ${ }^{30}$ Here we assumed a $\mu_{s}$ of $335 \mathrm{~cm}^{-1}$ at the laser excitation wavelength and ran a series of forward simulation based on initial guesses until the best fit is found. We have shown previously that the secondary filter effects (i.e., loss of the SHG signal) is negligible compared to the primary filter effect. $^{39}$ 
All the simulations were performed at the Wisconsin Center for High Throughput Computing at the University of Wisconsin-Madison and the values were stored in tables, permitting the image processing to be performed in custom MATLAB programs.

\subsubsection{SHG image-based metrics}

Multifractal analysis determines if a power-law scaling exists for various statistical moments at different scales, where monofractal and multifractal scaling behaviors are characterized by a single scaling exponential or a nonlinear function of the moments, respectively. ${ }^{40}$ In our specific case, a broader distribution of grayscale values (and feature sizes) would have a broader range of exponential values. The multifractal analysis was performed with the MATLAB tool in the signal-processing toolbox.

Entropy is a statistical measure of randomness that can be used to characterize the texture of the input image. In this context, entropy is defined as $-\operatorname{sum}[p . * \log 2(p)]$, where $p$ contains the normalized histogram count and was calculated with the MATLAB tool in the signal-processing toolbox.

For the maximum overlap discrete wavelet transform, the wavelet and scaling filter coefficients at level $j$ are computed by taking the inverse discrete Fourier transform (DFT) of a product of DFTs. This is performed using the MATLAB tool in the signal-processing toolbox.

\subsection{Statistical Analysis}

Separate one-way analyses of variance with Fisher's least significant difference (LSD) posthoc tests were performed comparing extracted average SHG conversion efficiencies and average $F_{\mathrm{SHG}} / B_{\mathrm{SHG}}$ creation ratios from each sample cohort: VP and AP wt and $\mathrm{Dcn}^{-/-}$. Each analysis compared the final extracted values from 9 to 12 total 3-D axial series from three randomly chosen fields of view from three unique mice from each cohort. The $p$-values $<0.05$ were considered statistically significant. Following the conclusion that Fisher's LSD posthoc tests found $p<0.05$ significance only in the AP WT versus $\mathrm{Dcn}^{-/-}$ samples for SHG conversion efficiencies and $F_{\mathrm{SHG}} / B_{\mathrm{SHG}}$ creation ratios (see Sec. 3.2), all further image analyses were performed solely on the AP WT versus Dcn ${ }^{-/-}$sample cohorts. For these, sample means testing were performed using two-tailed, two-sample equal variance Student's $t$-tests. All statistical analyses were completed using SAS (SAS Institute, Cary, North Carolina).

\section{Results}

\subsection{Collagen Fiber Morphology}

The left and right columns of Fig. 1 show representative SHG images of prostate tissue extracted from wt and Den null mice, respectively, of the AP (top) and VP (bottom) lobes. Prostate tissue from the wt mice appears to have predominately thicker, higher-ordered collagen fibers in the AP. In contrast, Dcn null prostate tissue consists of a mostly thin, smaller network of fibers. These differences are much less pronounced for the VP gland. This could be due to differences in the morphology of the two different lobes previously shown by histology. ${ }^{41,42}$ Specifically, the AP is composed of densely folded epithelium within a thick fibromuscular sheath, whereas the VP is composed of simple acini surrounded by a thin fibromuscular sheath

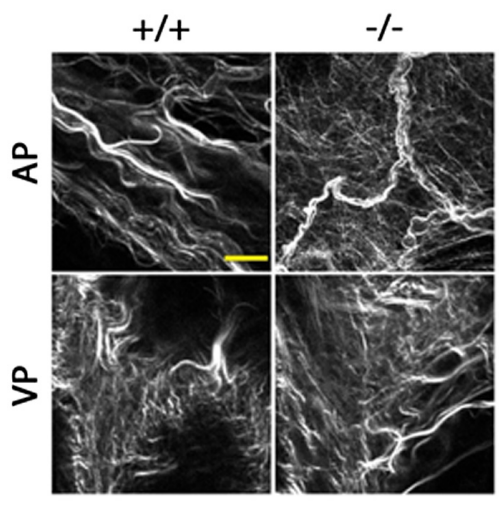

Fig. 1 Representative SHG optical images from wt and $\mathrm{Dcn}^{-/-}$adult $\mathrm{AP}$ and VP prostatic lobes. Scale bar $=10 \mu \mathrm{m}$.

enmeshed in a loose reticular stroma. Thus, given the intrinsic irregularity of fibrillar mesh-like structure of the VP, loss of Dcn may then have little effect on the collagen structure. In contrast, the highly fibrillar AP is significantly affected by Den loss.

Other than showing the similarity in the forward attenuation data (relative conversion efficiency) where both AP and VP are shown, we will focus our analyses on the AP glands as significant differences in the respective VP glands were not found (see Sec. 3.2). We note that there is no significant variation in collagen morphology across different sampling regions.

\subsection{SHG Forward Attenuation and Forward/ Backward Ratios}

\subsubsection{Conversion efficiency measurements by forward attenuation analysis}

Figure 2 shows the forward attenuation curves (SHG intensity versus depth) for the AP and VP of the wt and $\mathrm{Dcn}^{-/-}$prostates. First, we note that there are minimal differences between the VP glands of the tissues. This is generally consistent with the observations of the similar morphology of the wt and $\mathrm{Dcn}^{-/-}$in this gland, as shown in Fig. 1, where similar morphologies will have similar phase-matching conditions and similar SHG intensity. The response of the wt AP likely appears similar to both the VP glands due to the normalization process that is necessary to plot these data between different tissues on the same scale. As a result, tissues that have different SHG conversion efficiencies and scattering properties can have similar-looking attenuation curves, and as a consequence, this analysis is best for comparing the same type tissue (i.e., same gland) from normal and pathologic tissues. ${ }^{30}$ Here, the attenuation curve for the AP $\mathrm{Dcn}^{-/-}$decays much faster than the AP wt. Based on the underlying physics, this means that $\mathrm{Dcn}^{-/-}$has lower conversion efficiency which would correspond to either less collagen or less ordered collagen or thinner collagen bundles, all of which are suggested by the image data in Fig. 1.

We use the SHG MCML framework to extract the relative SHG conversion efficiencies of the normal and $\mathrm{Dcn}^{-/-} \mathrm{AP}$ glands, where the simulation includes both the primary $\left(335 \mathrm{~cm}^{-1}\right)$ and secondary filters $\left(445 \mathrm{~cm}^{-1}\right.$; although the former dominates the response). ${ }^{39}$ The data and best fit simulations are shown in Fig. 2(b). Based on the published values of the optical properties, ${ }^{36}$ we have extracted relative conversion efficiencies of $10.82 \pm 1.35$ and $4.78 \pm 1.29$ for the normal and $\mathrm{Dcn}^{-/-}$tissues, respectively. These are statistically different 

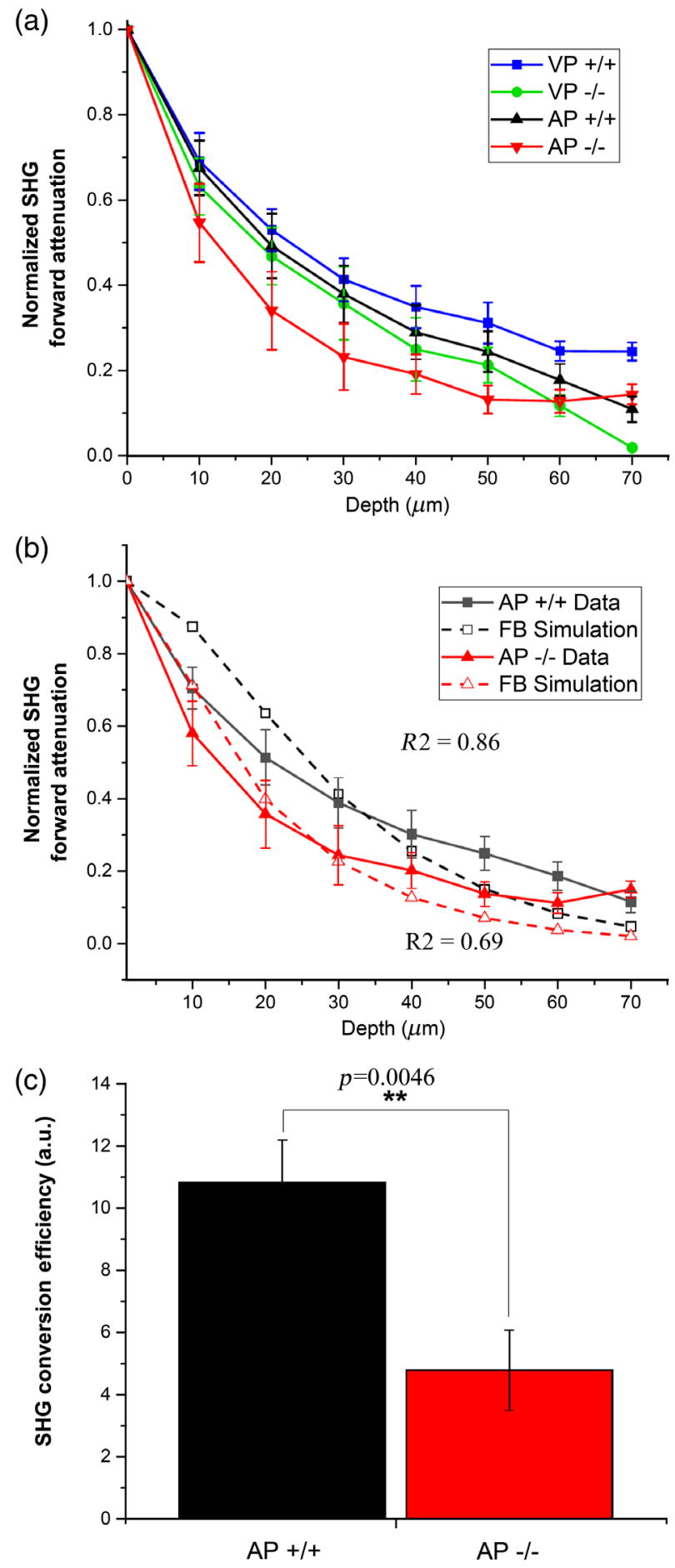

Fig. 2 (a) Forward SHG emission attenuation curves (intensity versus depth) for the four tissue types, normalized by peak power per series every $10 \mu \mathrm{m}$. Each line represents the mean \pm SEM of $n=3$. No significant change is detected in the VP lobes. (b) Best-fit Monte Carlo simulations to the experimental data of the $\mathrm{AP}^{+/+}$and $\mathrm{AP}^{-/-}$based on the primary and secondary filers and guesses to the conversion efficiency. The $R^{2}$ values between the data and best simulation are 0.86 and 0.69 for the wt and $\mathrm{Dcn}^{-/-}$, respectively. (c) Bar graph of the extracted SHG conversion efficiencies for the AP gland, where the $\mathrm{Dcn}^{-/-}$has significantly lower SHG conversion efficiencies compared to wt $(p=0.0046)$.
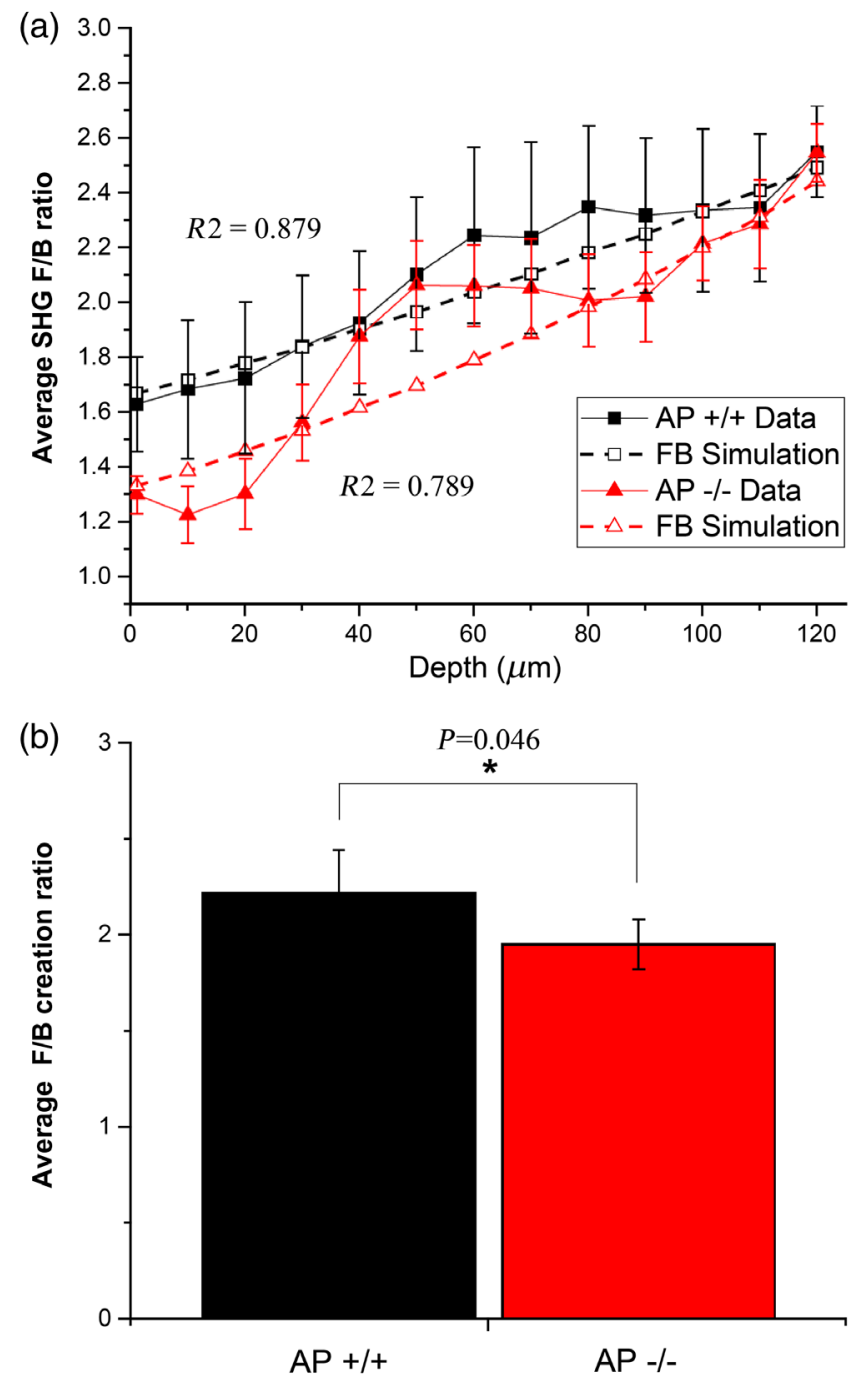

Fig. 3 (a) The measured forward/backward SHG as a function of depth (solid symbols and solid lines) for the $\mathrm{AP}^{+/+}$and $\mathrm{AP}^{-/-}$ where each line represents the mean \pm SEM of $n=3$ along with the best-fit simulation based on the reduced scattering coefficient at $445 \mathrm{~nm}$ (open symbols and lines). The $R^{2}$ values between the data and best simulation are 0.88 and 0.79 for the wt and $\mathrm{Dcn}^{-/-}$, respectively. The best-fit SHG emission directionality and significance $(p=0.046)$ are shown in (b). The results are consistent with the $\mathrm{Dcn}^{-/-}$having decreased fibril organization.

( $p=0.0046)$. Moreover, these values are consistent with the wt having a more ordered collagen structure and/or thicker collagen fibers, resulting in better phase matching and brighter SHG.

\subsubsection{Fibril size and distribution via SHG emission directionality}

The measured $F$ versus $B$ as function of depth response is used to extract the $F_{\mathrm{SHG}} / B_{\mathrm{SHG}}$ emission direction, which is a subresolution parameter that arises from the fibril size and packing relative to $\lambda_{\mathrm{SHG}}$. Figure 3 (a) shows the averaged response from three tissues for the wt and Dcn AP lobes. We note that the $\mathrm{Dcn}^{-/-} \mathrm{AP}$ lobe has a significantly lower measured F/B versus depth ratio compared to wt, which we have shown is reflective of lower fibril organization. To quantify this difference, we use the SHG MCML framework to decouple the initial emission directionality $F_{\mathrm{SHG}} / B_{\mathrm{SHG}}$ from the scattered component at 
$\lambda_{\mathrm{SHG}}$. The best fit simulations for the wt and $\mathrm{Dcn}^{-/-}$using published values for $\mu_{s}$ and $g^{30,36}$ are given in Fig. 3(a) $\left(R^{2}\right.$ values of 0.88 and 0.79 , respectively). We then extracted $F_{\text {SHG }} / B_{\text {SHG }}$ values of 3.35 versus 2.85 for the wt and $\mathrm{Dcn}^{-/-}$, respectively $(p=0.046)$. The lower value for the null is consistent with the structure having either smaller or more disordered fibrils relative to the wt. Thus, our SHG analysis is consistent with structural analyses of the null in other tissues (skin etc.) by SEM, which directly showed a more disordered fibril structure. ${ }^{23}$

\subsection{Image Analysis Features}

\subsubsection{Entropy and multifractal dimension}

To characterize the distribution of contrast levels from the collagen architecture between the wt and $\mathrm{Dcn}^{-/-} \mathrm{AP}$ gland tissues, we determined the image entropy as a statistical measure. Here the entropy ranges from 0 to 1 where higher levels correspond to a larger distribution of gray scale levels, where in this formalism the absolute values are calculated. Figure 4(a) shows the
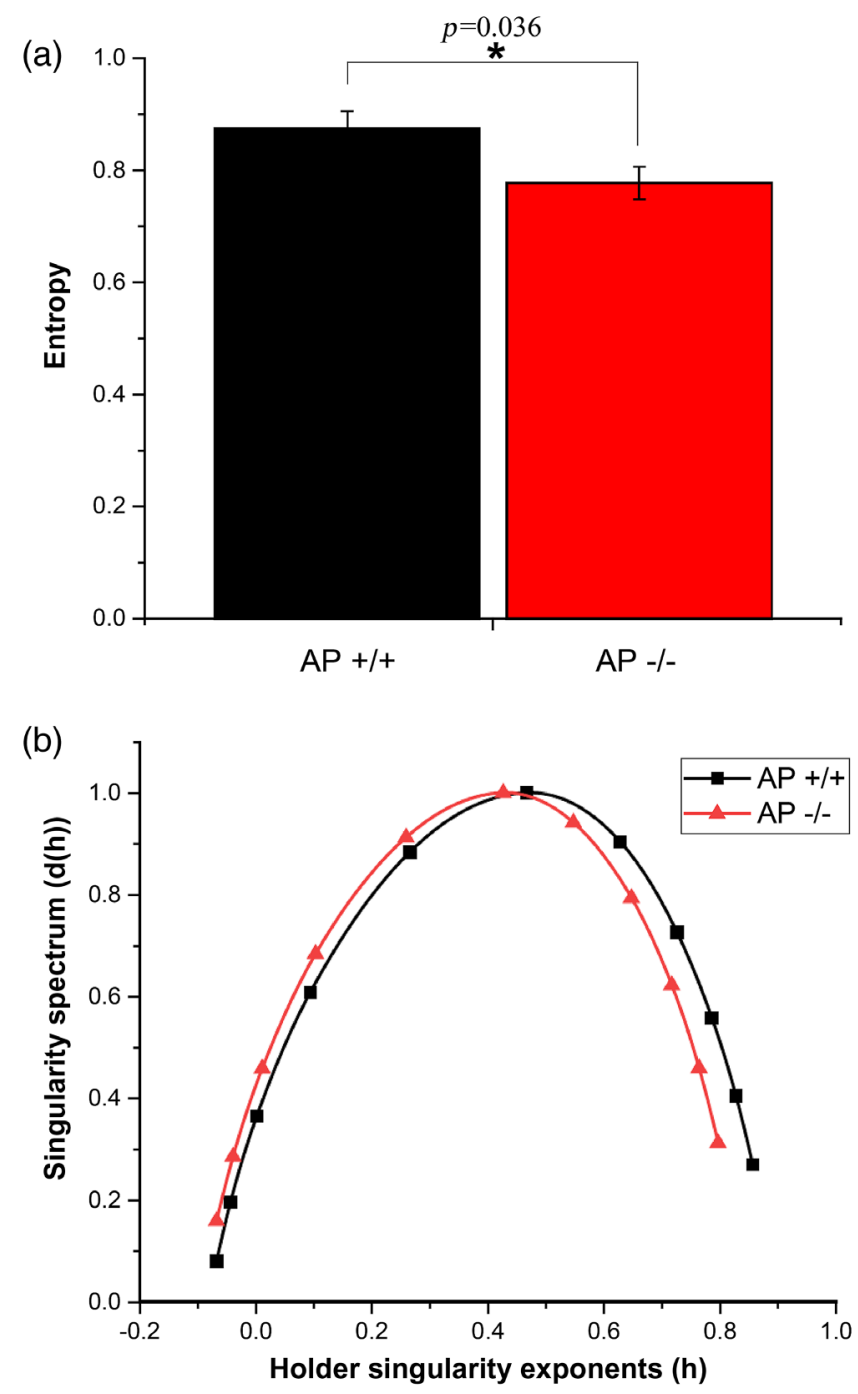

Fig. 4 (a) Entropy of the forward-directed $\mathrm{SHG}$ for the $\mathrm{AP}^{+/+}$and $\mathrm{AP}^{-/-}$, where the higher value for the $\mathrm{AP}^{+/+}$is consistent with larger structures $(p=0.036)$. (b) The multifractal dimension for the $\mathrm{AP}^{+/+}$ and $\mathrm{AP}^{-/-}$where the larger values for the $\mathrm{AP}^{+/+}$are further consistent with larger, more organized structures. extracted image entropy levels for the wt $(0.87 \pm 0.03)$ and $\mathrm{Dcn}^{-/-}(0.77 \pm 0.02)$, where the latter had significantly decreased entropy $(p=0.036)$. The larger entropy for the wt indicates that the SHG arises from a wider distribution of larger collagen structures. This is consistent with the higher $F_{\mathrm{SHG}} / B_{\mathrm{SHG}}$ values extracted for the wt [Fig. 3(b)], which arise from larger and more orderly distributed fibrils than those of the $\mathrm{Dcn}^{-/-}$.

We next used multifractal analysis to further probe the distribution of fibers in each tissue. Figure 4(b) shows the resulting multifractal analysis of the forward collected SHG for the wt and $\mathrm{Dcn}^{-/-}$tissues. Here, $d(h)$ is the normalized probability of the Holder singularity exponent $h$. We point out that the wt has a broader distribution and extends to higher values in the distribution. This is consistent with larger ordered structures in the fibrillar architecture in the wt and is further consistent with the entropy analysis. The respective multifractal distributions for the backward collected SHG are similar to the forward SHG (not shown).

\subsubsection{Quantification of coherence and effect on seg- mented image features}

Figure 5 shows representative $F$ and $B$ collected images for the wt and $\mathrm{Dcn}^{-/-}$from the AP glands. For both tissues, the forward collected images showed clear fibrillar behavior, whereas the backward collected images have many punctate or segmented spots mixed in with clear, contiguous fibers. However, these segmented fibers are much more evident in $\mathrm{Dcn}^{-/-}$. We have previously reported this phenomenon in other tissues and shown that it arises from destructive interference within the focal volume in the backward emitted $\mathrm{SHG}^{32,43}$ This arises because the coherence length of backward emitted SHG is shorter than the forward direction, as the phase mismatch $\Delta k$ is larger, where the coherence length is given as $2 \pi / \Delta k$. In this framework, more disordered fibrils, with larger phase mismatch, as those seen in the $\mathrm{Dcn}^{-/-}$, will lead to significant numbers of "segmented" fibers. (a)

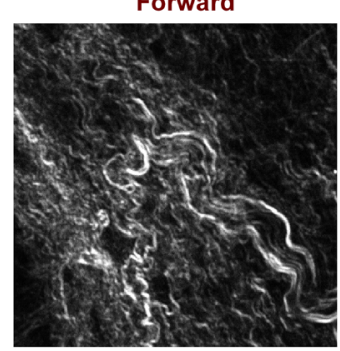

(b)

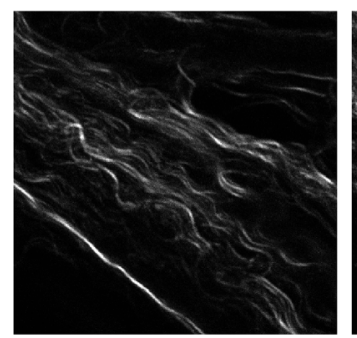

Backward

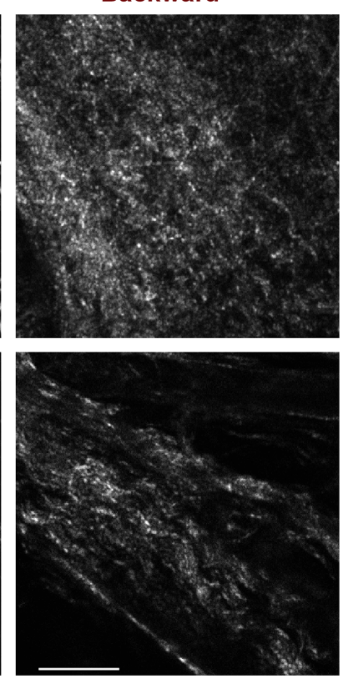

Fig. 5 Representative forward and backward collected SHG images of the $\mathrm{Dcn}^{-/-}$AP (a) and (b) wt AP. The segmented features in the backward channel arise from destructive interference from shorter coherence length structures, which are more pronounced in the $\mathrm{Dcn}^{-/-}$. Scale bar $=50 \mu \mathrm{m}$. 
We can use the extent of these segmented features to further compare the collagen architecture in wt and $\mathrm{Dcn}^{-/-} \mathrm{AP}$ glands. Our approach uses the maximal overlap discrete wavelet transform to probe the distribution of sizes of the features in the images. We have calculated the resulting energy coefficients from the transform in each level, where each scale corresponds to $2^{\hat{n}}$ pixels. The results for the forward and backward images comparing the wt and $\mathrm{Dcn}^{-/-}$are shown in Figs. 6(a) and 6(b), respectively, along with the statistical differences between the tissues. In the forward collected SHG, there are no significant differences between the tissues, although the lower scales have values $p \sim 0.1$ and higher $p$ values at larger scales. This corresponds to somewhat slightly dissimilar sizes of features in the tissues at small size and increasing similarity at larger-sized structures, comprising larger collections of fibers. This is consistent with the phase matching as small features (less than $\lambda_{\mathrm{SHG}}$ )
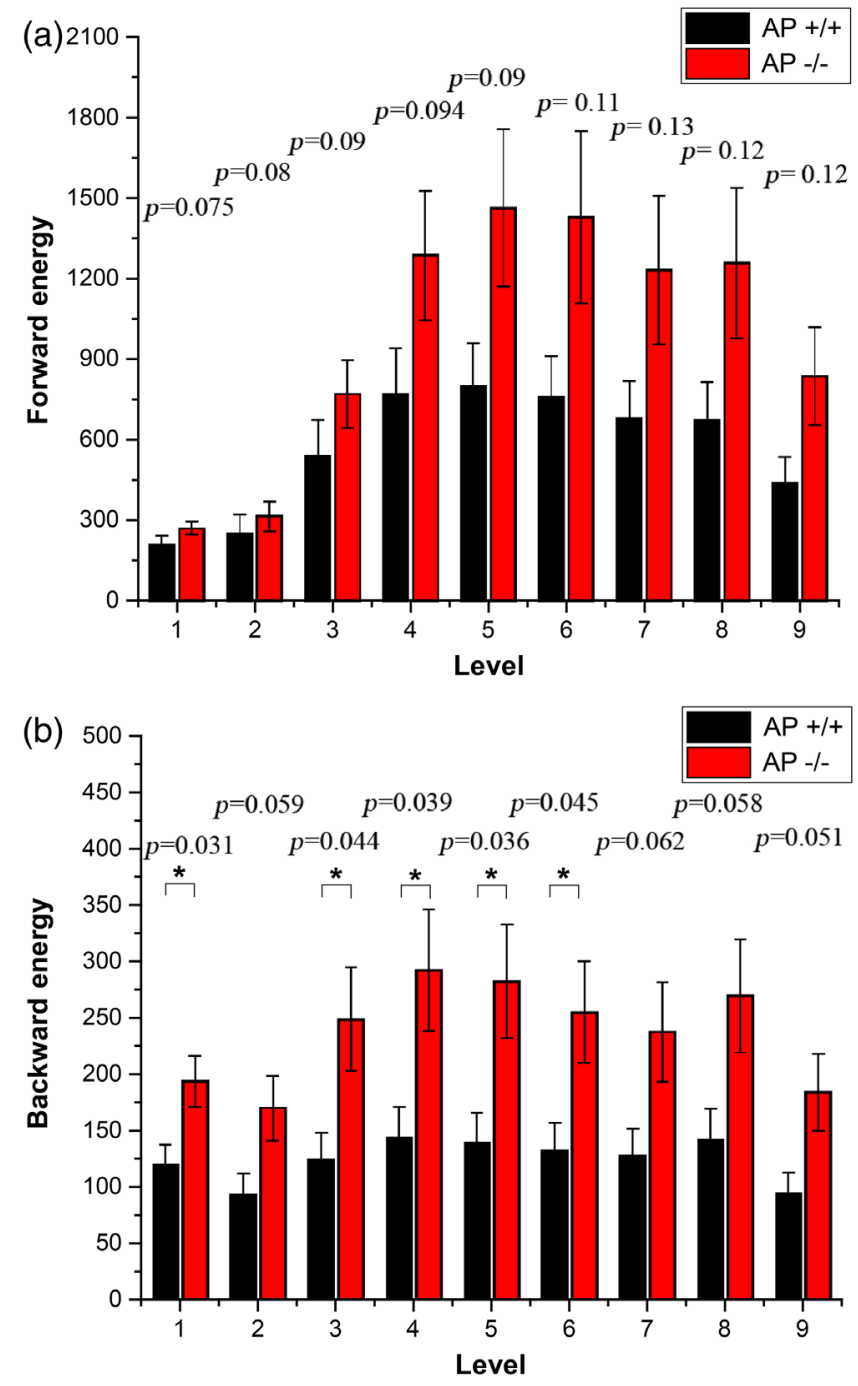

Fig. 6 Calculated energy coefficients and associated $p$ values from the maximum overlap discrete wavelet transform from the forward (a) and backward (b) SHG from the $\mathrm{AP}^{+/+}$and $\mathrm{AP}^{-/-}$glands. There are no statistical differences in the forward direction, where in contrast there are differences in the backward SHG between the $\mathrm{AP}^{+/+}$and $\mathrm{AP}^{-/-}$at low levels, corresponding to small differences in the distribution of small structures in the tissues, consistent with the increased segmented features in the $\mathrm{Dcn}^{-/-}$in Fig. 5(a). result in both $F_{\mathrm{SHG}}$ and $B_{\mathrm{SHG}}$, whereas larger features result only in forward emitted SHG.

Statistical differences are seen in the analysis of the backward collected data. The differences at low and medium scales are highly significant, where these diminish at larger scales. We stress that smaller sizes in the backward channel result from smaller features (and smaller $F_{\mathrm{SHG}} / B_{\mathrm{SHG}}$ values), whereas larger structures arise from multiple scattering of the forward SHG, rather than backward emitted SHG (i.e., $B_{\mathrm{SHG}}$ ). We further note that the segmented features arise from subresolution fibril sizes (and packing therein), but they can be discerned by SHG through the differences in coherence length. ${ }^{31}$ In this case, we next note that this analysis completely agrees with the emission direction analysis in the prior section, where the $\mathrm{Dcn}^{-/-}$had lower $F_{\mathrm{SHG}} / B_{\mathrm{SHG}}$ values, corresponding to less ordered/smaller fibrils. We also note that while we have seen these segmented features in other tissues ${ }^{32,43}$ the $\mathrm{Dcn}^{-/-}$prostate is the most striking example to date.

\section{Discussion and Conclusions}

Our goal for this study was to establish a direct link between Den and the organization of the prostatic collagen matrix. SHG imaging and quantification techniques such as emission directionality $\left(F_{\mathrm{SHG}} / \mathrm{B}_{\mathrm{SHG}}\right)$, SHG conversion efficiency, and image feature analyses (including wavelet transforms, entropy determinations, and fractal dimension analysis) probed statistically significant structural differences (both fibril and fiber) in prostate collagen assembly in $\mathrm{Dcn}^{-/-}$vis-à-vis wt mice. Importantly, all the measures were self-consistent with the conclusion of the $\mathrm{Dcn}^{-/-}$having smaller, more disorganized fibrils. We note that this was achieved with a small cohort of three wt and $\mathrm{Dcn}^{-/-}$mice each. This most likely occurred because there was extensive collagen remodeling associated with the loss of decorin coupled with the high sensitivity/specificity of SHG for imaging these alterations. This is also in line with our previous use of SHG for many applications. ${ }^{28-30,37,39}$

The results are all consistent with the structural biology of Den in other tissues, where the $\mathrm{Dcn}^{-/-}$has more randomly ordered fibrils but has not been shown in prostate. ${ }^{13,23}$ Additionally, our results confirm and expands the well-known biological function of decorin during development, that is, its ability to "decorate" collagen at crucial points. ${ }^{44}$ Indeed, decorin binds near the $\mathrm{C}$ terminus of collagen type I in a zone that coincides with the $\mathrm{c} 1$ band of the collagen fibril D-period. This location is very close to one of the major intermolecular crosslinking sites of collagen heterotrimers, suggesting that lack of decorin would interfere with collagen fibril stability. These aspects have been previously studied by ultrastructural techniques, ${ }^{14}$ but here are measured on the SHG microscope. In particular, the $\mathrm{F}_{\mathrm{SHG}} / \mathrm{B}_{\mathrm{SHG}}$ emission ratio is directly linked to the fibril size and packing, and the lower value for the $\mathrm{Dcn}^{-/-}$prostate is consistent with the structural biology findings.

We previously characterized the role of Den in development and morphogenesis of the mouse prostate. ${ }^{19}$ Our studies showed that loss of Den significantly increased epithelial proliferation and decreased branching morphogenesis in the AP. Similar trends that did not reach statistical significance were observed in the VP. The divergence of these findings in the AP and VP is echoed by the observations reported showing collagen disorganization to be more pronounced in the AP, as shown by all the SHG metrics here. A possible explanation for this is differing functional redundancy for Den in the AP and VP. Biglycan is 
a proteoglycan highly related to decorin (70\% shared similarity). Like Dcn, biglycan appears to play a role in the organization of collagen fibrils and there is evidence for some functional overlap between these two proteoglycans. ${ }^{24}$ Our preliminary studies have shown that biglycan is expressed in the developing prostate but differential expression in the lobes of the developing prostate has not yet been studied (unpublished observations).

Decorin and its loss have been implicated in several prostatic pathologies. For example, Dcn has been implicated as a tumor suppressor in prostate cancer ${ }^{14}$ where observational studies indicate a general trend for decreased Den expression. ${ }^{45,46}$ The antitumor activity has previously been attributed to the effects on signaling pathways $;{ }^{47}$ however, disruption of collagen organization could be a contributing factor. Recent studies have demonstrated that perturbations in collagen organization play an important role in several cancers. ${ }^{33,48-51}$ Similarly, collagen remodeling has been shown to occur in prostate cancer, where there is a nonuniform swelling and disorganization of collagen fibers. ${ }^{52,53}$ Our analysis here provides more in-depth structural characterization of the prostate that can contribute to better understanding of the alterations in cancer. It is tempting to speculate that the loss of Dcn expression or function could result in a discoordination of collagen organization and growth regulation resulting in breakdown of the normal homeostatic mechanisms that inhibit development and progression of neoplasia. Collagen turnover may also be related to the prevalence of inflammation in the adult prostate. ${ }^{54}$ In sum, we believe this work may be useful in making connections between collagen structural changes and prostatic pathologies that may have direct future clinical impact.

\section{Disclosures}

The authors declare no competing financial interests.

\section{Acknowledgments}

PJC acknowledges support under NIH 1R01CA206561-01. WB and PJC acknowledge the UW Carbone Cancer Center. The original research on decorin was supported in part by $\mathrm{NIH}$ Grant No. RO1 CA-39481 (RVI). The Monte Carlo simulations were all performed at the Wisconsin Center for High Throughput Computing at the University of WisconsinMadison.

\section{References}

1. S. D. Chipman et al., "Defective pro alpha 2(I) collagen synthesis in a recessive mutation in mice: a model of human osteogenesis imperfecta," Proc. Natl. Acad. Sci. U. S. A. 90, 1701-1705 (1993).

2. J. Baum and B. Brodsky, "Folding of peptide models of collagen and misfolding in disease," Curr. Opin. Struct. Biol. 9, 122-128 (1999).

3. N. P. Camacho et al., "The material basis for reduced mechanical properties in oim mice bones," J. Bone Miner. Res. 14, 264-272 (1999).

4. R. J. Wenstrup et al., "Type V collagen controls the initiation of collagen fibril assembly," J. Biol. Chem. 279, 53331-53337 (2004).

5. M. Sun et al., "Collagen V is a dominant regulator of collagen fibrillogenesis: dysfunctional regulation of structure and function in a cornealstroma-specific Col5a1-null mouse model,' J. Cell Sci. 124, 4096-4105 (2011).

6. C. Ricciardelli and R. J. Rodgers, "Extracellular matrix of ovarian tumors," Semin. Reprod. Med. 24, 270-282 (2006).

7. S. H. Barsky et al., "Increased content of type V Collagen in desmoplasia of human breast carcinoma," Am. J. Pathol. 108, 276-283 (1982).

8. J. M. Seyer, E. T. Hutcheson, and A. H. Kang, "Collagen polymorphism in idiopathic chronic pulmonary fibrosis," J. Clin. Invest. 57, 1498-1507 (1976).
9. X. Chen et al., "Second harmonic generation microscopy for quantitative analysis of collagen fibrillar structure," Nat. Protoc. 7, 654-669 (2012).

10. T. M. Bauman et al., "Characterization of fibrillar collagens and extracellular matrix of glandular benign prostatic hyperplasia nodules," PLoS One 9, e109102 (2014).

11. L. Yuting et al., "Microscale characterization of prostate biopsies tissues using optical coherence elastography and second harmonic generation imaging," Lab Invest. 98, 380-390 (2018).

12. Y. Ling et al., "Second harmonic generation (SHG) imaging of cancer heterogeneity in ultrasound guided biopsies of prostate in men suspected with prostate cancer," J. Biophotonics 10, 911-918 (2017).

13. K. G. Danielson et al., "Targeted disruption of decorin leads to abnormal collagen fibril morphology and skin fragility," J. Cell Biol. 136, 729-743 (1997).

14. I. J. Edwards, "Proteoglycans in prostate cancer," Nat. Rev. Urol. 9, 196-206 (2012).

15. R. V. Iozzo et al., "Cooperative action of germ-line mutations in decorin and p53 accelerates lymphoma tumorigenesis," Proc. Natl. Acad. Sci. U. S. A. 96, 3092-3097 (1999).

16. X. Bi et al., "Genetic deficiency of decorin causes intestinal tumor formation through disruption of intestinal cell maturation," Carcinogenesis 29, 1435-1440 (2008).

17. X. Bi et al., "Decorin-mediated inhibition of colorectal cancer growth and migration is associated with E-cadherin in vitro and in mice," Carcinogenesis 33, 326-330 (2012).

18. X. Bi et al., "Oncogenic activin $\mathrm{C}$ interacts with decorin in colorectal cancer in vivo and in vitro," Mol. Carcinog. 55, 1786-1795 (2016).

19. M. Montano et al., "Dual regulation of decorin by androgen and Hedgehog signaling during prostate morphogenesis," Dev. Dyn. 247, 679-685 (2018).

20. M. Montano and W. Bushman, "Morphoregulatory pathways in prostate ductal development," Dev. Dyn. 246, 89-99 (2017).

21. J. P. Orgel et al., "Decorin core protein (decoron) shape complements collagen fibril surface structure and mediates its binding," PLoS One 4, e7028 (2009).

22. C. C. Reed and R. V. Iozzo, "The role of decorin in collagen fibrillogenesis and skin homeostasis," Glycoconj. J. 19, 249-255 (2002).

23. C. Ruhland et al., "The glycosaminoglycan chain of decorin plays an important role in collagen fibril formation at the early stages of fibrillogenesis," FEBS J. 274, 4246-4255 (2007).

24. A. Corsi et al., "Phenotypic effects of biglycan deficiency are linked to collagen fibril abnormalities, are synergized by decorin deficiency, and mimic Ehlers-Danlos-like changes in bone and other connective tissues," J. Bone Miner. Res. 17, 1180-1189 (2002).

25. S. P. Reese et al., "Effects of decorin proteoglycan on fibrillogenesis, ultrastructure, and mechanics of type I collagen gels," Matrix Biol. 32, 414-423 (2013).

26. K. A. Robinson et al., "Decorin and biglycan are necessary for maintaining collagen fibril structure, fiber realignment, and mechanical properties of mature tendons," Matrix Biol. 64, 81-93 (2017).

27. P. Campagnola, "Second harmonic generation imaging microscopy: applications to diseases diagnostics," Anal. Chem. 83, 3224-3231 (2011).

28. K. Tilbury et al., "Second harmonic generation microscopy analysis of extracellular matrix changes in human idiopathic pulmonary fibrosis," J. Biomed. Opt. 19, 086014 (2014).

29. K. B. Tilbury et al., "Stromal alterations in ovarian cancers via wavelength dependent second harmonic generation microscopy and optical scattering," BMC Cancer 17, 102 (2017).

30. R. Lacomb, O. Nadiarnykh, and P. J. Campagnola, "Quantitative SHG imaging of the diseased state osteogenesis imperfecta: experiment and simulation," Biophys. J. 94, 4504-4514 (2008).

31. R. Lacomb et al., "Phase matching considerations in second harmonic generation from tissues: effects on emission directionality, conversion efficiency and observed morphology," Opt. Commun. 281, 1823-1832 (2008).

32. O. Nadiarnykh et al., "Second harmonic generation imaging microscopy studies of osteogenesis imperfecta," J. Biomed. Opt. 12, 051805 (2007).

33. B. Wen et al., "3D texture analysis for classification of second harmonic generation images of human ovarian cancer," Sci. Rep. 6, 35734 (2016). 
34. L. Wang, S. L. Jacques, and L. Zheng, "MCML-Monte Carlo modeling of light transport in multi-layered tissues," Comput. Methods Prog. Biomed. 47, 131-146 (1995).

35. G. Hall, K. W. Eliceiri, and P. J. Campagnola, "Simultaneous determination of the second-harmonic generation emission directionality and reduced scattering coefficient from three-dimensional imaging of thick tissues," J. Biomed. Opt. 18, 116008 (2013).

36. S. L. Jacques, "Optical properties of biological tissues: a review," Phys. Med. Biol. 58, R37-R61 (2013).

37. O. Nadiarnykh et al., "Alterations of the extracellular matrix in ovarian cancer studied by Second Harmonic Generation imaging microscopy," BMC Cancer 10, 94 (2010).

38. G. Hall et al., "Experimental and simulation study of the wavelength dependent second harmonic generation of collagen in scattering tissues," Opt. Lett. 39, 1897-1900 (2014).

39. R. LaComb et al., "Quantitative SHG imaging and modeling of the optical clearing mechanism in striated muscle and tendon," J. Biomed. Opt. 13, 021109 (2008).

40. R. Lopes and N. Betrouni, "Fractal and multifractal analysis: a review," Med. Image Anal. 13, 634-649 (2009).

41. M. Ittmann, "Anatomy and histology of the human and murine prostate," Cold Spring Harb Perspect. Med. 8, a030346 (2018).

42. D. S. Oliveira et al., "The mouse prostate: a basic anatomical and histological guideline," Bosn. J. Basic Med. Sci. 16, 8-13 (2016).

43. O. Nadiarnykh et al., "Coherent and incoherent SHG in fibrillar cellulose matrices," Opt. Express 15, 3348-3360 (2007).

44. D. R. Keene et al., "Decorin binds near the $\mathrm{C}$ terminus of type I collagen," J. Biol. Chem. 275, 21801-21804 (2000).

45. A. Henke et al., "Stromal expression of decorin, Semaphorin6D, SPARC, Sprouty1 and Tsukushi in developing prostate and decreased levels of decorin in prostate cancer," PLoS One 7, e42516 (2012).

46. A. V. Suhovskih et al., "Proteoglycan expression in normal human prostate tissue and prostate cancer," ISRN Oncol. 2013, 680136 (2013).

47. Y. Hu et al., "Decorin suppresses prostate tumor growth through inhibition of epidermal growth factor and androgen receptor pathways," Neoplasia 11, 1042-1053 (2009).

48. M. W. Conklin et al., "Aligned collagen is a prognostic signature for survival in human breast carcinoma," Am. J. Pathol. 178, 1221-1232 (2011).

49. C. R. Drifka et al., "Highly aligned stromal collagen is a negative prognostic factor following pancreatic ductal adenocarcinoma resection," Oncotarget 7(46), 76197-76213 (2016).
50. R. Cicchi et al., "Multidimensional non-linear laser imaging of basal cell carcinoma," Opt. Express 15, 10135-10148 (2007).

51. E. Brown et al., "Dynamic imaging of collagen and its modulation in tumors in vivo using second-harmonic generation," Nat. Med. 9, 796-800 (2003).

52. C. Morrison, J. Thornhill, and E. Gaffney, "The connective tissue framework in the normal prostate, BPH and prostate cancer: analysis by scanning electron microscopy after cellular digestion," Urol. Res. 28, 304-307 (2000).

53. J. Tang et al., "Tissue elasticity displayed by elastography and its correlation with the characteristics of collagen type I and type III in prostatic stroma," Asian J. Androl. 16, 305-308 (2014).

54. L. Wong, P. R. Hutson, and W. Bushman, "Resolution of chronic bacterial-induced prostatic inflammation reverses established fibrosis," Prostate 75, 23-32 (2015).

Kirby R. Campbell was a PhD student in the Biomedical Engineering Department at the University of Wisconsin and obtained his degree in 2017. Currently, he is a postdoctoral fellow at St. Jude Children's Research Hospital in Memphis.

Rajeev Chaudhary was a PhD student in the biomedical engineering department at the University of Wisconsin and obtained his degree in 2017. Currently, he works for Berkeley Lighting.

Monica Montano was a PhD student in the cellular and molecular pathology program at the University of Wisconsin and obtained her degree in 2017. Currently, she works in the science policy sector.

Renato V. lozzo is the Gonzalo Aponte Endowed Chair Professor in the Pathology Department at Thomas Jefferson University and is a leader in the matrix biology field as well as the editor-in-chief of Matrix Biology.

Wade A. Bushman is a physician scientist at the University of Wisconsin in the department of urology and specializes in the research and treatment of benign prostate disorders.

Paul J. Campagnola is a professor in the biomedical engineering and medical physics departments at the University of Wisconsin. He specializes in developing nonlinear optical approaches to study changes in the extracellular matrix in diseased states. 